TRANSACTIONS OF THE

AMERICAN MATHEMATICAL SOCIETY

Volume 358, Number 4 , Pages 1421-1434

S 0002-9947(05)04083-3

Article electronically published on September 9, 2005

\title{
COVERING A COMPACT SET IN A BANACH SPACE BY AN OPERATOR RANGE OF A BANACH SPACE WITH BASIS
}

\author{
V. P. FONF, W. B. JOHNSON, A. M. PLICHKO, AND V. V. SHEVCHYK
}

\begin{abstract}
A Banach space $X$ has the approximation property if and only if every compact set in $X$ is in the range of a one-to-one bounded linear operator from a space that has a Schauder basis. Characterizations are given for $\mathcal{L}_{p}$ spaces and quotients of $\mathcal{L}_{p}$ spaces in terms of covering compact sets in $X$ by operator ranges from $\mathcal{L}_{p}$ spaces. A Banach space $X$ is a $\mathcal{L}_{1}$ space if and only if every compact set in $X$ is contained in the closed convex symmetric hull of a basic sequence which converges to zero.
\end{abstract}

\section{INTRODUCTION}

A Banach space $Y$ is said to have the approximation property (AP) if for every compact $K \subset Y$ and every $\varepsilon>0$ there exists a linear operator $T$ of finite rank such that $\|T x-x\|<\varepsilon, x \in K$. If, in addition, $T$ can be chosen with $\|T\| \leq \lambda$, for some constant $\lambda$ independent of $K$ and $\varepsilon$, then $Y$ is said to have the bounded approximation property (BAP). To complete the line (AP) $\Longleftarrow(\mathrm{BAP})$ denote the property of a Banach space to have a basis by (BP). Thus we have

$$
(\mathrm{AP}) \Longleftarrow(\mathrm{BAP}) \Longleftarrow(\mathrm{BP}) \text {. }
$$

All these properties are different (see [FJ] and $[\underline{\mathrm{Sz}}$ ). However, the following interesting connection between the BAP and the BP was established in [P1] and JRZ.

Theorem 1.1. A separable Banach space $E$ has the BAP iff $E$ is isomorphic to a complemented subspace of a Banach space with basis.

Actually the space with basis in this theorem may be chosen to be universal for all spaces with the BAP (see $[\mathrm{P} 2]$ and $[\mathrm{Sc}$ ).

In this paper we establish the following connection between the AP and the BP.

Theorem 1.2. For a Banach space $X$ the following properties are equivalent:

(i) $X$ has the $A P$.

(ii) For each compact subset $K$ of $X$ there exist a Banach space $Y$ with basis and a one-to-one linear operator $T: Y \rightarrow X$ such that $T(Y) \supset K$.

(iii) There exists a reflexive Banach space $R$ with basis and with unconditional finite-dimensional decomposition such that for each compact $K \subset B_{X}$ and for each

Received by the editors September 7, 2001 and, in revised form, July 9, 2002.

2000 Mathematics Subject Classification. Primary 46B28; Secondary 46B15, 46B25, 46B50.

The second author was supported in part by NSF DMS-9900185, DMS-0200690, Texas Advanced Research Program 010366-0033-20013, and the U.S.-Israel Binational Science Foundation.

The third author was supported in part by the DAAD Foundation. 
$\gamma>0$ there is a compact one-to-one operator $T: R \rightarrow X$ with $T\left(B_{R}\right) \supset K$ and $\|T\| \leq 1+\gamma$.

The condition that $T$ is one-to-one is essential. Indeed, it is not difficult to show that each compact set in every Banach space may be covered by an operator range of $\ell_{1}$.

In Section 2 we prove that the space $R$ in (iii) may be chosen to be universal for all spaces with AP (see Theorem 2.1).

Section 3 contains the "converse result" and a characterization of the AP in terms of the existence of an $M$-basis with special properties. Here we recall that a sequence $\left\{x_{n}, x_{n}^{*}\right\}_{n=1}^{\infty}$ in $X \times X^{*}$ is biorthogonal provided $x_{n}^{*}\left(x_{m}\right)=\delta_{n m}$ for all $n$ and $m$. A biorthogonal sequence $\left\{x_{n}, x_{n}^{*}\right\}_{n=1}^{\infty}$ is fundamental provided the linear span of $\left\{x_{n}\right\}_{n=1}^{\infty}$ is dense in $X$ and is total provided $\left\{x_{n}^{*}\right\}_{n=1}^{\infty}$ separates the points of $X$. An $M$-basis is a fundamental and total biorthogonal sequence. A basis can then be thought of as a biorthogonal sequence $\left\{x_{n}, x_{n}^{*}\right\}_{n=1}^{\infty}$ such that for every $x$ in $X$, the series $\sum x_{n}^{*}(x) x_{n}$ converges to $x$, so that a basis is an $M$-basis.

With the help of the results proved in Sections 2 and 3 we get (in Section (3) a factorization theorem for compact operators acting into spaces with the AP (see Theorem 3.3 below).

In Section 4 we deal with $\mathcal{L}_{p}$-spaces. We characterize these spaces in terms of covering compact sets and in terms of the factorization of compact operators. We also classify those Banach spaces $X$ in which every compact set is contained in the closed, convex, symmetric hull of a basic sequence which converges to zero.

We use standard Banach space geometry notation (see [LT]). If $X$ is a Banach space and $A \subset X$, then $[A]$ is the closed linear span of $A$. Let $H \subset X$ be a closed, convex, symmetric, and bounded subset of a Banach space $X$. Then it is wellknown that the linear submanifold $\operatorname{span} H$ becomes a Banach space if we introduce in it the norm with the unit ball $H$. We denote this later Banach space by $E_{H}$.

The authors thank J. Lindenstrauss for a fruitful discussion and for a series of suggestions that improved the paper.

\section{Covering a COMpact Set in a Banach space with the AP}

Theorem 2.1. There exists a reflexive Banach space $R$ with basis and with unconditional finite-dimensional decomposition such that for each Banach space $X$ with $A P$, for each compact $K \subset B_{X}$ and for each $\gamma>0$ there is a compact one-to-one operator $T: R \rightarrow X$ with $T\left(B_{R}\right) \supset K$ and $\|T\| \leq 1+\gamma$.

We start with some auxiliary results.

Lemma 2.2. Let $\left\{E_{i}\right\}$ be a sequence of finite-dimensional subspaces of a Banach space $X$ and let $\left\{C_{i}\right\}$ be a sequence of linear operators on $X$ with $C_{i}(X) \subset E_{i}, i=$ $1,2, \ldots$. Let $Y$ be the set of all sequences $y=\left(x_{i}\right)_{i=1}^{\infty}, x_{i} \in E_{i}$, such that the series $\sum x_{i}$ converges, and let $Z$ be the set of all vectors $x \in X$ such that $x=\sum_{i=1}^{\infty} C_{i} x$. Then $Y$ is a Banach space with monotone finite-dimensional decomposition (FDD) in the norm $\|y\|^{\prime}=\sup \left\{\left\|\sum_{i=1}^{n} x_{i}\right\|: n=1,2, \ldots\right\}$ and $Z$ is a Banach space in the norm $\|x\|=\sup _{n}\left\|\sum_{i=1}^{n} C_{i} x\right\|, x=\sum_{i=1}^{\infty} C_{i} x$. Moreover the operator $J: Z \rightarrow Y$ defined by $J x=\left(C_{i} x\right), x \in Z$, is an isometry.

Proof. First we prove that $Y$ is complete. Let $\left\{y_{m}\right\} \subset Y, y_{m}=\left(x_{i}^{m}\right)$, be such that $\lim _{p, q \rightarrow \infty}\left\|y_{p}-y_{q}\right\|^{\prime}=0$. Clearly, for each $i$ the sequence $\left\{x_{i}^{m}\right\}_{m=1}^{\infty}$ converges to 
some $x_{i} \in E_{i}$. First we check that the series $\sum x_{i}$ converges. Fix $\varepsilon>0$ and find an integer $s$ such that for each $p, q \geq s$ the inequality $\left\|y_{p}-y_{q}\right\|^{\prime}<\varepsilon$ holds. Let $r$ be such that $\left\|\sum_{i=l}^{k} x_{i}^{s}\right\|<\varepsilon$ for each $k>l>r$. We have for $k>l>r$

$$
\left\|\sum_{i=l}^{k} x_{i}\right\|=\lim _{p}\left\|\sum_{i=l}^{k} x_{i}^{p}\right\| \leq \lim _{p}\left\|\sum_{i=l}^{k} x_{i}^{s}\right\|+\lim _{p}\left\|\sum_{i=l}^{k}\left(x_{i}^{p}-x_{i}^{s}\right)\right\|<3 \varepsilon .
$$

Thus $y=\left(x_{i}\right) \in Y$. Finally we show that $\lim y_{m}=y$. Let $\varepsilon$ and $s$ be as above. Then for each $n$ and for each $p, q>s$ we have $\left\|\sum_{i=1}^{n}\left(x_{i}^{p}-x_{i}^{q}\right)\right\| \leq \varepsilon$. By pushing $q$ to infinity we have $\left\|\sum_{i=1}^{n}\left(x_{i}^{p}-x_{i}\right)\right\| \leq \varepsilon$, for each $n$ and for each $p>s$. By taking the supremum we have for each $p>s,\left\|y_{p}-y\right\|^{\prime} \leq \varepsilon$.

Next we prove that $Z$ is complete. Put $B_{n}=\sum_{i=1}^{n} C_{i}, n=1,2, \ldots, B_{0}=I d_{X}$. It is well known that the set $W$ of all vectors $x \in X$ such that the $\lim _{n} B_{n} x$ exists is a Banach space in the norm

$$
\|\mid x\|=\sup \left\{\left\|B_{n} x\right\|: n=0,1,2, \ldots\right\} .
$$

Clearly, $Z \subset W$. We check that $Z$ is closed in $W$. Let $\left\{x_{m}\right\} \subset Z$, let \|\|$\cdot\|\|-\lim x_{m}=$ $x, x \in W$, and let $\varepsilon>0$. Take $m$ so large that $\left\|\mid x_{m}-x\right\| \|=\sup \left\{\left\|B_{n} x_{m}-B_{n} x\right\|\right.$ : $n=0,1,2, \ldots\}<\varepsilon$. Clearly, $\left\|x_{m}-x\right\|<\varepsilon$, too. Next by using that $x_{m} \in Z$, find an $n_{0}$ such that $\left\|x_{m}-B_{n} x_{m}\right\|<\varepsilon$ holds for each $n>n_{0}$. We have for $n>n_{0}$

$$
\left\|x-B_{n} x\right\| \leq\left\|x-x_{m}\right\|+\left\|x_{m}-B_{n} x_{m}\right\|+\left\|B_{n} x_{m}-B_{n} x\right\|<3 \varepsilon
$$

which proves that $\lim _{n} B_{n} x=x$, i.e. $x \in Z$.

The remaining part of the lemma is clear.

Remark 2.3. If for each $i, \operatorname{dim} E_{i}=1$, then the space $Y$ has a monotone basis.

Lemma 2.4. Let $E$ be a Banach space with monotone $F D D$ and let $K \subset B_{E}$ be compact. Then for every $\varepsilon>0$ there is a compact one-to-one operator $A: E \rightarrow E$ with $A\left(B_{E}\right) \supset K$ and $\|A\| \leq 1+\varepsilon$.

Proof. Let $S_{n}, n=1,2, \ldots$, be the partial sum operators associated with a given FDD $E=\sum E_{i}$. Take $\delta>0$ with $(1+\delta)^{2}<1+\varepsilon$ and let a sequence $\left\{\varepsilon_{i}\right\}_{i=1}^{\infty}$ of positive numbers be such that $\sum \varepsilon_{i}<\delta / 2$. By using the compactness of $K$, find an increasing sequence $\left\{n_{i}\right\}$ of integers such that

$$
\sup \left\{\left\|S_{n_{i+1}} y-S_{n_{i}} y\right\|: y \in K\right\}<\varepsilon_{i}^{2}, \quad i=1,2, \ldots
$$

Put

$$
Q_{0}=S_{n_{1}}, Q_{i}=S_{n_{i+1}}-S_{n_{i}}, i=1,2, \ldots, A_{1}=\sum_{i=0}^{\infty} \varepsilon_{i} Q_{i}, \varepsilon_{0}=1
$$

Clearly, $A_{1}$ is a compact one-to-one operator with $\left\|A_{1}\right\| \leq 1+\delta$. If $y \in K$, then the series $\sum_{i=0}^{\infty}\left(1 / \varepsilon_{i}\right) Q_{i} y$ converges absolutely (say to $z$ ). It is not difficult to check that $\|z\| \leq 1+\delta$ and that $A_{1} z=y$. Thus $A_{1}\left((1+\delta) B_{E}\right) \supset K$. Put $A=(1+\delta) A_{1}$.

Remark 2.5. Let $H=\left\{y \in B_{E}: y=\sum_{i=0}^{\infty} y_{i}, y_{i} \in Q_{i}(E),\left\|y_{i}\right\| \leq \varepsilon_{i}, i=0,1, \ldots\right\}$. It is clear from the proof of the lemma that $A(H) \supset K$.

Lemma 2.6. Let $L, M \subset Y$ be closed subspaces of a separable Banach space $Y$ such that $\operatorname{dim} M=\infty$ and $L \cap M=\{0\}$, and let $F \subset Y$ be compact. Then there is an automorphism $D: Y \rightarrow Y$ such that $L \cap D(F) \subset\{0\}$ and $\left.D\right|_{M}=I d_{M}$. 
Proof. Let $\left\{f_{i}\right\} \subset B_{M^{\perp}}$ be a sequence which is total over $L$ and put

$$
V=\operatorname{cl} \operatorname{co}\left(B_{L} \cup \pm F\right) \text {. }
$$

The linear manifold $\operatorname{span} V$, being the image of $E_{V}$ under the injection of $E_{V}$ into $Y$, is an operator range, and clearly $\operatorname{codim} \operatorname{span} V=\infty$. By [Fo1], Lemma 3, there is a minimal (even basic) sequence $\left\{t_{i}\right\} \subset Y$ with $\left\|t_{i}\right\|<2^{-i-1}$ and such that $\sum a_{i} t_{i} \notin \operatorname{span} V$ for each non-zero bounded sequence of numbers $\left\{a_{i}\right\}$. Define an operator $C: Y \rightarrow Y$ by $C x=x+\sum f_{i}(x) t_{i}$. Clearly, $C$ is an isomorphism of $Y$ onto $Y$ and $\left.C\right|_{M}=I d_{M}$. We check that $C(L) \cap F \subset\{0\}$. Let $u \in C(L) \cap F$. Then for some $z \in L$ we have $u=z+\sum f_{i}(z) t_{i} \in F$ and hence $\sum f_{i}(z) t_{i}=u-z \in \operatorname{span} V$. By the choice of $\left\{t_{i}\right\}$ we have $f_{i}(z)=0, i=1,2, \ldots$, and, since $\left\{f_{i}\right\}$ is total over $L$, it follows that $z=0$. Thus $u=0$ which proves that $C(L) \cap F \subset\{0\}$ and hence $L \cap C^{-1}(F) \subset\{0\}$. Put $D=C^{-1}$. This completes the proof.

The following lemma is known $\mathrm{P} 1$. We give a proof for the sake of completeness.

Lemma 2.7. For any finite-dimensional Banach space $L$ and for any $\varepsilon>0$ there is a finite-dimensional Banach space $M$ with monotone basis which contains a $(1+\varepsilon)$ isomorphic copy of $L$ as a $(1+\varepsilon)$-complemented subspace.

Proof. Let $\left\{x_{i}\right\}_{i=1}^{n}$ and $\left\{x_{i}^{*}\right\}_{i=1}^{n}$ be an Auerbach system for $L, n=\operatorname{dim} L$. Take an integer $p$ with $n / p<\varepsilon$ and define linear operators $U_{i}$ in $L$ as follows:

$$
U_{i} x=\frac{1}{p} x_{r}^{*}(x) x_{r}, i=n l+r, 1 \leq r \leq n, l=0,1, \ldots, p-1 .
$$

Put $m=p n$. Clearly, $\sum_{j=1}^{m} U_{j}=I d_{L}$. For $i=n l+r$ we have the estimate

$$
\left\|\sum_{j=1}^{i} U_{j}\right\|=\left\|\sum_{j=1}^{n l} U_{j}+\sum_{j=n l+1}^{n l+r} U_{j}\right\| \leq l / p+r / p \leq(p-1+n) / p \leq 1+\varepsilon .
$$

Let $E_{i}=\operatorname{span}\left\{y_{i}\right\},\left\|y_{i}\right\|=1, i=1, \ldots, m$, be the (1-dimensional) range of $U_{i}$ (actually $y_{i}=x_{r}$ for $i=n l+r, 1 \leq r \leq n, l=0,1, \ldots, p-1$ ). Define $M$ as the space of all $m$-dimensional vectors $y=\left(a_{i}\right)_{i=1}^{m}$ with the norm $\|y\|=\max _{1 \leq q \leq m}\left\|\sum_{i=1}^{q} a_{i} y_{i}\right\|$. It is clear that the vectors $e_{i}=(0, \ldots, 1, \ldots, 0)$ ( 1 stands in the $i$-th place), $i=1, \ldots, m$, form a monotone basis of $M$. Define an operator $A: L \rightarrow M$ by $A x=\left(a_{i}\right)_{i=1}^{m}$, where $a_{i}$ is defined by $U_{i} x=a_{i} y_{i}, i=1, \ldots, m$. Put $L_{1}=A(L)$. From the construction it is clear that $L$ is $(1+\varepsilon)$-isomorphic to $L_{1}$. Finally define an operator $P: M \rightarrow L_{1}$ as follows:

$$
P\left(a_{i}\right)=A\left(\sum a_{i} y_{i}\right),\left(a_{i}\right) \in M .
$$

It is not difficult to see that $P$ is a projection and $\|P\| \leq 1+\varepsilon$.

Proof of Theorem 2.1. Take $\varepsilon>0$ and a sequence of positive numbers $\left\{\varepsilon_{i}\right\}_{i=0}^{\infty}, \varepsilon_{0}=$ 1 , with $\sum_{i=1}^{\infty} \varepsilon_{i}<\varepsilon$.

Let $K \subset B_{X}$ be a compact subset of a Banach space $X$ with the AP. It is well known (see [LT, Proposition 1.e.2) that there is a sequence $\left\{x_{i}\right\} \subset X$ with $\lim x_{i}=0$ and such that $\operatorname{cl} \operatorname{co}\left\{x_{i}\right\} \supset K$. By a slight modification of the proof given in [LT, Proposition 1.e.2, we can get that $\left\{x_{i}\right\} \subset(1+\varepsilon) B_{X}$. Let a sequence of positive numbers $\left\{\lambda_{i}\right\}$ be such that $\lim \lambda_{i}=\infty,\left\{\lambda_{i} x_{i}\right\} \subset(1+\varepsilon) B_{X}$, and $\lim \lambda_{i} x_{i}=0$. Put $K_{0}=\operatorname{cl} \operatorname{co}\left\{ \pm \lambda_{i} x_{i}\right\}$. 
Since $X$ has the AP there is a sequence $\left\{B_{n}\right\}$ of finite-dimensional operators in $X$ such that

$$
\sup \left\{\left\|B_{n} x-x\right\|: x \in K_{0}\right\}<\varepsilon_{n}, n=1,2, \ldots .
$$

Put $C_{1}=B_{1}, C_{n+1}=B_{n+1}-B_{n}, n=1,2, \ldots$. Then for each $x \in K_{0}, x=$ $\sum_{n=1}^{\infty} C_{n} x$ and

$$
\sup _{1 \leq n<\infty} \sup \left\{\left\|\sum_{i=1}^{n} C_{i} x\right\|: x \in K_{0}\right\} \leq 1+2 \varepsilon .
$$

Next for each $i$ put $E_{i}=C_{i}(X)$ and define the spaces $Y$ and $Z$ as in Lemma 2.2 , In a natural way we can consider $K_{0}$ as a subset of $Z$. Thus, from Theorem 2.1 we have

$$
\sup \left\{\mid\|x\| \|: x \in K_{0}\right\} \leq 1+2 \varepsilon .
$$

Define a summation operator $B: Y \rightarrow X$ as follows:

$$
B\left(x_{i}\right)=\sum_{i=1}^{\infty} x_{i}, \quad\left(x_{i}\right) \in Y .
$$

Clearly, $\|B\|=1$. Put $L=\operatorname{Ker} B$.

Since $\lambda_{i} \rightarrow \infty$ and since $K_{0}$ is bounded in $Z$, it easily follows that $K$ considered as a subset of $Z$ is compact. Let $J: Z \rightarrow Y$ be the natural embedding, i.e. $J x=\left(V_{m} x\right)$. By Lemma 2.2, $J$ is an isometry and, in particular, $M=J(Z)$ is a (closed) subspace of $Y$. A simple verification shows that $L \cap M=\{0\}$.

Now we pass to the space $R$. We use for $R$ the space constructed in [J]. Recall the construction of $R$. Let $\mathcal{A}_{n}$ be the family of all $n$-dimensional Banach spaces with monotone basis, i.e. an $n$-dimensional space $M \in \mathcal{A}_{n}$ iff there is a basis $\left\{x_{i}\right\}_{i=1}^{n}$ of $M$ such that all partial sum operators have norm 1. Let $\left\{M_{n, i}\right\}_{i=1}^{\infty}$ be a dense (in the Banach-Mazur metric) sequence in $\mathcal{A}_{n}, n=1,2, \ldots$. Enumerate the set $\left\{M_{n, i}\right\}_{n, i=1}^{\infty}$ into a sequence $\left\{M_{n}\right\}_{n=1}^{\infty}$ and put

$$
R=\left(\sum_{n=1}^{\infty} M_{n}\right)_{\ell_{2}}
$$

Clearly, $R$ has a (monotone) basis and an unconditional finite-dimensional decomposition.

Next, by using Lemma 2.4 find a compact one-to-one operator $A: Y \rightarrow Y$, $A=\sum_{i=0}^{\infty} \varepsilon_{i} Q_{i},\|A\| \leq(1+2 \varepsilon)^{2}$, such that $A\left(B_{Y}\right) \supset J\left(K_{0}\right)$. Put $L_{j}=Q_{j}(Y), j=$ $0,1, \ldots$, and by Lemma 2.7 find for each $j$ a space $M_{n_{j}}$ such that $M_{n_{j}}=V_{j}+W_{j}$, where $V_{j}$ is $\left(1+\varepsilon_{j}\right)$-isomorphic to $L_{j}$ and $\left(1+\varepsilon_{j}\right)$-complemented in $M_{n_{j}}$. Let $T_{j}: V_{j} \rightarrow L_{j}$ be an isomorphism satisfying $\left\|T_{j}\right\|\left\|T_{j}^{-1}\right\| \leq 1+\varepsilon_{j}, j=0,1, \ldots$.

Now we define a compact 1-1 operator $S: R \rightarrow Y$. We first define $S$ on the subspace $V=\left(\sum_{j=0}^{\infty} V_{j}\right)_{\ell_{2}} \subset R$ as follows: $S\left(x_{j}\right)=\sum_{j=0}^{\infty} \varepsilon_{j} T_{j} x_{j}, x_{j} \in V_{j}, j=$ $0,1, \ldots$ It is not difficult to see that $\left.S\right|_{V}$ is a compact 1-1 operator, and $\left\|\left.S\right|_{V}\right\| \leq 1+\varepsilon$ (recall that $\left.\varepsilon_{0}=1\right)$. By $[\mathrm{Pl}$ (see also [Fo1]) there is a (closed) infinite-dimensional subspace $E \subset Y$ with $E \cap S(V)=\{0\}$. Next define $S$ on the remaining part of $R$, i.e., on the subspace $W=\left(\sum_{n \neq n_{j}} M_{n}\right)_{\ell_{2}} \oplus\left(\sum_{j=0}^{\infty} W_{j}\right)_{\ell_{2}}$ in such a way that $\left.S\right|_{W}$ is a one-to-one compact operator into $E$ with norm so small that $\|S\| \leq 1+2 \varepsilon$.

Put $F=A S\left(B_{R}\right)$. It is clear from the construction of $S$ and Remark 2.5 that $F \supset K$. 
By using Lemma 2.6 find an automorphism $D: Y \rightarrow Y,\|D\| \leq 1+\varepsilon$ such that $L \cap D(F)=\{0\}$ and $D_{\left.\right|_{M}}=I d_{M}$. Put $T=B D A S$. Then $T: R \rightarrow X$ is a compact one-to-one operator and $T\left(B_{R}\right) \supset K$. From the construction it is clear that $\|T\| \leq(1+2 \varepsilon)^{3}$. Clearly, for $\varepsilon>0$ small enough we get $\|T\| \leq 1+\gamma$ which completes the proof.

Remark 2.8. Let $U$ be a Banach space with basis that contains (isomorphically) each Banach space with basis as a complemented subspace (for constructions of such spaces see $\mathrm{P} 2$ and $[\mathrm{Sc}]$ ). We show that such a space $U$ may be used in Theorem 2.1 instead of $R$. First, by an obvious modification of the proof of Theorem 2.1, we can get that $T^{-1}(K)$ is compact. Clearly, $R \subset U$. Let $P: U \rightarrow R$ be a projection from $U$ onto $R$ and let $A_{1}: U \rightarrow U$ be a compact one-to-one operator such that $A_{1}\left(B_{U}\right) \supset T^{-1}(K)$ (see Lemma 2.4). Put $F_{1}=\mathrm{cl} A_{1}\left(B_{U}\right), \quad L_{1}=\operatorname{Ker} P$ and let $D_{1}: U \rightarrow U$ be an automorphism such that $L_{1} \cap D_{1}\left(F_{1}\right)=\{0\}$ and $\left.D_{1}\right|_{R}=I d_{R}$ (see Lemma 2.6). Then the operator $T_{1}=T P D A: U \rightarrow X$ is a one-to-one compact operator such that $T_{1}\left(B_{U}\right) \supset K$.

Remark 2.9. Let $\left\{K_{n}\right\}_{n=1}^{\infty}$ be a sequence of compact sets in a Banach space $X$ with the AP. Put $K=\bigcup_{n=1}^{\infty}\left(n \max \left\{\|x\|: x \in K_{n}\right\}\right)^{-1} K_{n}$. Clearly, $K \cup\{0\}$ is compact and, by Theorem 2.1 there is a compact operator $T: R \rightarrow X$ with $T\left(B_{R}\right) \supset K$. In particular, $T(R) \supset \bigcup_{n=1}^{\infty} K_{n}$.

Lemma 2.10. Let $R$ be a reflexive Banach space with basis and let $T: R \rightarrow X$ be a 1-1 linear operator from $R$ into a separable Banach space $X$. Then there are a basis $\left\{y_{i}\right\}$ of $R$ and a norming $M$-basis $\left\{x_{i}\right\}$ of $X$ such that $\left\{T y_{i}\right\} \subset\left\{x_{i}\right\}$.

Proof. Recall that a subspace $Y$ of $X^{*}$ is called norming provided the expression \|\|$x\|\|:=\sup \{f(x): f \in Y,\|f\| \leq 1\}$ defines an equivalent norm on $X$. An $M$-basis $\left\{x_{n}, x_{n}^{*}\right\}_{n=1}^{\infty}$ is norming provided $\left[x_{n}^{*}\right]$ is norming.

First we assume that $[T(R)]=X$. Let $F \subset X^{*}$ be a countable-dimensional norming subspace of $X^{*}$. Since $R$ is reflexive and $T$ is $1-1$, it follows that $T^{*}(F)$ is a countable-dimensional dense subspace of $R^{*}$. Clearly, $R^{*}$ has a basis. Now we use the following result from $[\mathrm{T}]$ :

Let $E$ be a Banach space with basis and let $L \subset E$ be a countable-dimensional dense subspace of $E$. Then there is a basis $\left\{z_{n}\right\}$ of $E$ with $\operatorname{span}\left\{z_{n}\right\}=L$.

Take $E=R^{*}$ and $L=T^{*}(F)$ and let $\left\{g_{n}\right\}$ be a basis of $R^{*}$ with $\operatorname{span}\left\{g_{n}\right\}=$ $T^{*}(F)$. Let $\left\{e_{n}\right\}$ be the vectors in $R$ which are biorthogonal to $\left\{g_{n}\right\}$. It is clear that $x_{n}=T e_{n}, n=1,2, \ldots$, is a norming $M$-basis of $X$ with the desired properties.

If $X_{1}=[T(R)]$ is a proper subspace of $X$ we first construct as above a norming $M$-basis of $X_{1}$ and then extend it (see [Fo2]) to a norming $M$-basis of $X$. The proof is complete.

Corollary 2.11. Let $X$ be a separable Banach space with the AP. Then for each compact set $K \subset X$ there is a norming $M$-basis $\left\{x_{i}\right\}$ in $X$ with biorthogonal functionals $\left\{x_{i}^{*}\right\}$ such that for each $x \in K$ we have

$$
x=\sum_{i=1}^{\infty} x_{i}^{*}(x) x_{i} \text { and } \sup _{n} \sup _{x \in K}\left\|\sum_{i=1}^{n} x_{i}^{*}(x) x_{i}\right\|<\infty .
$$

Proof. By Theorem 2.1 there is a linear 1-1 operator $T: R \rightarrow X$ from a reflexive space $R$ with basis into $X$ such that $T\left(B_{R}\right) \supset K$. Apply Lemma 2.10 and complete the proof. 
Remark 2.12. Let $\mu$ be a probability measure on a separable Banach space $X$. An $M$-basis $\left\{x_{i}\right\}$ for $X$ (with biorthogonal functionals $\left\{x_{i}^{*}\right\}$ ) is called a stochastic basis for $(X, \mu)$ if $\mu\left\{x \in X: x=\sum x^{*}(x) x_{i}\right\}=1$ (see He). It follows from Remark 2.9 and Corollary 2.11 that if a Banach space $X$ has the AP, then for each probability measure $\mu,(X, \mu)$ has a stochastic basis. For more about stochastic bases and the stochastic approximation property, see [FJPP], which is a follow-up to this paper.

Remark 2.13. In view of Theorem 2.1 the following question is natural: is it possible to substitute for the space $R$ in Theorem 2.1 a space with an unconditional basis? The following example shows that the answer is negative.

By GL, for each integer $n$ there is a finite-dimensional space $E_{n}$ such that for each Banach space $Y$ with 1-unconditional basis and for any two operators $A: E_{n} \rightarrow Y$ and $B: Y \rightarrow E_{n}$ with $B A=I d_{E_{n}}$, the inequality $\|A\|\|B\| \geq n$ holds. Put $X=\left(\sum E_{n}\right)_{\ell_{2}}$ and let $X_{1}$ be a copy of $X$. Let $T: X \rightarrow X_{1}$ be a linear operator such that $\left.T\right|_{E_{n}}=I d_{E_{n}} / \sqrt{n}, n=1,2, \ldots$. Set $K=T\left(B_{X}\right)$. Clearly, $K$ is a compact set. We check that $K$ cannot be covered by a one-to-one operator range of a Banach space with unconditional basis. Suppose the contrary, i.e., for some Banach space $Y$ with unconditional basis and for some operator $B: Y \rightarrow X_{1}, K$ is a subset of $B(Y)$. Consider the operator $A=B^{-1} T: X \rightarrow Y$. A simple verification shows that $A$ is a closed operator, so $A$ is bounded by the closed graph theorem. Clearly, $T=B A$ and $\left.\sqrt{n} T\right|_{E_{n}}=\left.I d\right|_{E_{n}}=\left.\sqrt{n} B A\right|_{E_{n}}$ for each $n$. By taking into account the property of the space $E_{n}$ we deduce that $\sqrt{n}\left\|\left.A\right|_{E_{n}}\right\|\|B\| \geq n$. In particular, $\|A\| \geq \sqrt{n} /\|B\|$ for each $n$ which contradicts the boundedness of $A$.

\section{The CONVERSE RESUlt}

Theorem 3.1. Let $X$ be a Banach space such that for each compact $H \subset X$ there are a Banach space $Y$ with the AP and a one-to-one operator $T: Y \rightarrow X$ such that $T Y \supset H$. Then $X$ has the AP.

Proof. Fix a compact $K \subset X$ and $\varepsilon>0$. We shall construct a finite-dimensional operator $C$ in $X$ such that $\sup \{\|C x-x\|: x \in K\}<\varepsilon$.

By a well-known result (see [LT, Proposition 1.e.2) there is a sequence $\left\{x_{i}\right\} \subset X$ such that $\lim x_{i}=0$ and that $\operatorname{cl} \operatorname{co}\left\{x_{i}\right\} \supset K$. Take a sequence of numbers $\left\{\lambda_{i}\right\}$ with $\lim \lambda_{i}=\infty$ and such that $\lim \lambda_{i} x_{i}=0$ and then put $H=\operatorname{cl} \operatorname{co}\left\{ \pm \lambda_{i} x_{i}\right\}$. Let $A: E_{H} \rightarrow X$ be the natural injection (recall that $E_{H}$ is the Banach space $\operatorname{span} H$ with the unit ball $H$ ). Let $T: Y \rightarrow X$ be a one-to-one linear operator from a Banach space $Y$ with the AP such that $T(Y) \supset H$. A simple verification shows that the operator $T^{-1} A: E_{H} \rightarrow Y$ is closed and by the closed graph theorem it is bounded. In particular, the set $T^{-1}(H)$ is bounded. From $\lim \lambda_{i}=\infty$ it easily follows that the set $F=T^{-1}(K)$ is compact. Next by using the AP of $Y$ find a finite-dimensional operator $B: Y \rightarrow Y$,

$$
B y=\sum_{i=1}^{n} f_{i}(y) e_{i}, \quad f_{i} \in Y^{*}, e_{i} \in Y, y \in Y,
$$

such that $\sup \{\|B y-y\|: y \in F\}<\varepsilon / 2\|T\|$.

Recall that the $b w^{*}$-topology on $Y^{*}$ is just the topology of uniform convergence on the compact subsets of $Y$. By the Krein-Smulian theorem the $b w^{*}$-closure of each convex subset of $Y^{*}$ coincides with its $w^{*}$-closure. In particular, $b w^{*}-\operatorname{cl} T^{*}\left(X^{*}\right)=$ $w^{*}-\operatorname{cl} T^{*}\left(X^{*}\right)$. Since $T$ is one-to-one it follows that $w^{*}-\operatorname{cl} T^{*}\left(X^{*}\right)=Y^{*}$ and hence 
$b w^{*}-\operatorname{cl} T^{*}\left(X^{*}\right)=Y^{*}$. The last equality allows us to find for each $i, 1 \leq i \leq n$, a linear functional $g_{i} \in T^{*}\left(X^{*}\right)$ such that

$$
\sup \left\{\left|g_{i}(y)-f_{i}(y)\right|: y \in F\right\}<\varepsilon /\left(2 n\left\|e_{i}\right\|\|T\|\right) .
$$

Take $h_{i} \in X^{*}$ such that $T^{*} h_{i}=g_{i}, i=1, \ldots, n$, and define an operator $C: X \rightarrow X$ by

$$
C x=\sum_{i=1}^{n} h_{i}(x) T e_{i}, \quad x \in X .
$$

We check that $C$ is as desired. Take $x \in K$, put $y=T^{-1} x \in F$, and write

$$
\begin{aligned}
\|C x-x\| & =\left\|\sum_{i=1}^{n} h_{i}(x) T e_{i}-x\right\|=\left\|T\left(\sum_{i=1}^{n} h_{i}(x) e_{i}-T^{-1} x\right)\right\| \\
& \leq\|T\|\left\|\sum_{i=1}^{n} g_{i}(y)-y\right\|=\|T\|\left\|\sum_{i=1}^{n} f_{i}(y)-y+\sum_{i=1}^{n}\left(g_{i}(y)-f_{i}(y)\right) e_{i}\right\| \\
& \leq\|T\|\left(\varepsilon / 2\|T\|+\sum_{i=1}^{n} \mid g_{i}(y)-f_{i}(y)\left\|e_{i}\right\|\right)<\varepsilon .
\end{aligned}
$$

The proof is complete.

Alternate proof. By a theorem of Grothendieck (see Theorem 1.e.4 in [LT]), it is enough to verify that an arbitrary compact operator $S$ from an arbitrary Banach space $Z$ into $X$ is (uniformly) approximable by finite-dimensional operators. The operator $S$ factors compactly through some Banach space $W$ ( $W$ can even be taken reflexive; see, e.g., $[\mathrm{F})$; that is, there are compact operators $A: Z \rightarrow W, D: W \rightarrow$ $X$ so that $D A=S$. Let $T$ be a one-to-one operator from some space $Y$ which has the AP into $X$ so that $T Y$ contains the closure of $D\left(B_{W}\right)$. The linear mapping $T^{-1} D$ is closed, hence is a bounded linear operator. Moreover, $T^{-1} D A: Z \rightarrow Y$ is a compact operator into a space with the AP, hence is approximable by finitedimensional operators, whence $S=T\left(T^{-1} D A\right)$ is also approximable by finitedimensional operators.

Corollary 3.2. For a Banach space $X$ the following assertions are equivalent:

(i) $X$ has the $A P$.

(ii) Any compact set $K \subset X$ may be covered by a 1-1 operator range of a Banach space with basis.

(iii) For any compact set $K \subset X$ there is an $M$-basic sequence $\left\{x_{i}\right\}$ in $X$ (with biorthogonal functionals $\left.\left\{x_{i}^{*}\right\}\right)$ such that $x=\sum x_{i}^{*}(x) x_{i}$ for each $x \in K$.

With the help of the above results we get the following factorization

Theorem 3.3. For a Banach space $X$ the following assertions are equivalent:

(i) $X$ has the AP.

(ii) For each Banach space $Z$ and for each compact operator $A: Z \rightarrow X$ there are compact operators $B: Z \rightarrow R$ and $T: R \rightarrow X$ such that $T$ is one-to-one and that $A=T B$. (Here $R=\left(\sum_{n=1}^{\infty} M_{n}\right)_{\ell_{2}}$ is the space from Theorem 2.1].)

(iii) For each Banach space $Z$ and for each compact operator $A: Z \rightarrow X$ there are a Banach space $Y$ with $A P$ and compact operators $B: Z \rightarrow Y$ and $T: Y \rightarrow X$ such that $A=T B$. 
Proof. (i) $\Longrightarrow$ (ii). Put $K=\operatorname{cl} A\left(B_{Z}\right)$ and as it was done in the proofs of Theorems 2.1 and 3.1, find $\left\{x_{i}\right\}_{i=1}^{\infty} \subset X, \lim _{i} x_{i}=0$ with cl $\operatorname{co}\left\{x_{i}\right\}_{i=1}^{\infty} \supset K$ and a sequence of numbers $\left\{\lambda_{i}\right\}_{i=1}^{\infty}, \lim _{i} \lambda_{i}=\infty$ such that $\lim _{i} \lambda_{i} x_{i}=0$. Put $K_{1}=\operatorname{cl} \operatorname{co}\left\{\lambda_{i} x_{i}\right\}_{i=1}^{\infty}$ and by using Theorem 2.1 find a one-to-one compact operator $T: R \rightarrow X T\left(a B_{R}\right) \supset$ $K_{1}$ for some $a>0$. Put $B=T^{-1} A$ and check that $B$ is a compact operator. Indeed, since $T^{-1}\left(K_{1}\right)$ is bounded it follows that $T^{-1}(K)$ is compact. But $B\left(B_{Z}\right)=$ $T^{-1} A\left(B_{Z}\right) \subset T^{-1}(K)$ which proves that $B$ is a compact operator and completes the proof of (i) $\Longrightarrow$ (ii).

(ii) $\Longrightarrow$ (iii). Obvious.

(iii) $\Longrightarrow$ (i). This is immediate from the result of Grothendieck (Theorem 1.e.4 in [LT]), used in the alternate proof of Theorem 3.1.

\section{Covering COMPACT SETS IN $\mathcal{L}_{p}$-SPACES AND FACTORIZING COMPACT OPERATORS INTO $\mathcal{L}_{p}$-SPACES}

As we already mentioned in the Introduction, any compact subset of any Banach space may be covered by an operator range of $\ell_{1}$. In this section we prove that every compact subset of a Banach space $X$ may be covered by a one-to-one range of $\ell_{1}$ if and only if $X$ is an $\mathcal{L}_{1}$-space (actually we prove a much stronger result; see Theorem 4.7 below). Also we get a characterization of $\mathcal{L}_{p}$-spaces in terms of covering compact sets and in terms of the factorization of compact operators.

We start with with two auxiliary results.

Lemma 4.1. For each natural number $n$ there is $m(n)<\infty$ so that if $E$ is an $n$-dimensional space which is $K$-isomorphic to a $K$-complemented subspace of $L_{p}$, $1 \leq p \leq \infty$, and $E$ has a basis with constant $K$, then $E \oplus \ell_{p}^{m(n)}$ is $f(K)$-isomorphic to $\ell_{p}^{n+m(n)}$.

Proof. By [PR], $E$ is $2 K$-isomorphic to a $2 K$-complemented subspace of $\ell_{p}^{m}$, where $m$ depends only on $n$. Then by BDGJN], $E \oplus_{p} \ell_{p}^{m n}$ is $f(K)$-isomorphic to $\ell_{p}^{(m+1) n}$.

The next lemma very slightly improves the results in [JRZ and [NW] that a separable $\mathcal{L}_{p}$ space has a basis with certain desirable properties.

Lemma 4.2. Let $X$ be a separable $\mathcal{L}_{p}$ space, $1 \leq p \leq \infty$. Then $X$ has a basis $\left\{x_{n}\right\}_{n=1}^{\infty}$ which satisfies

$$
\sup _{1 \leq n<m<\infty} d\left(\ell_{p}^{m-n}, \operatorname{span}\left\{x_{n}, x_{n+1}, \ldots, x_{m-1}\right\}\right)<\infty .
$$

Proof. Note that by Lemma 4.1 it is enough to show that for any sequence $0=$ $n_{1}<n_{2}<\ldots$, there is a basis $\left\{x_{n}\right\}_{n=1}^{\infty}$ for $X$ and a constant $K$ so that for all $k=1,2, \ldots,\left\{x_{i}\right\}_{i=n_{k}+1}^{n_{k+1}}$ is $K$-equivalent to the unit vector basis for $\ell_{p}^{n_{k+1}-n_{k}}$.

First we indicate how to get such a basis when $p<\infty$. In this case $X$ has a complemented subspace which is isomorphic to $\ell_{p}$, and hence $X$ is isomorphic to $X \oplus \ell_{p}$ since for some $Y$ we have $X \sim Y \oplus \ell_{p} \sim Y \oplus \ell_{p} \oplus \ell_{p} \sim X \oplus \ell_{p}$. Let $\left\{y_{n}\right\}_{n=1}^{\infty}$ be a basis for $X$ and let $\left\{e_{n}\right\}_{n=1}^{\infty}$ be the unit vector basis for $\ell_{p}$. Make a basis for $X \oplus \ell_{p}$ by interlacing these bases for $X$ and $\ell_{p}$, i.e., use $\left\{y_{1} \oplus 0,0 \oplus e_{1}, 0 \oplus\right.$ $\left.e_{2}, \ldots, 0 \oplus e_{m_{1}}, y_{2} \oplus 0,0 \oplus e_{m_{1}+1}, 0 \oplus e_{m_{1}+2}, \ldots, 0 \oplus e_{m_{2}}, \ldots\right\}$. As long as $m_{k}$ grows fast enough, this will be a basis that has the desired property.

The same argument works when $X$ is a separable $\mathcal{L}_{\infty}$ space which contains a (necessarily complemented) isomorphic copy of $c_{0}$, but to do the general case, one 
needs to "localize" the construction. Again, let $\left\{y_{n}\right\}_{n=1}^{\infty}$ be a basis for $X$. Let $\epsilon>0 ; \epsilon=1$ is fine for our purposes. For appropriate $m_{1}$, take a sequence $\left\{e_{i}^{1}\right\}_{i=1}^{m_{1}}$ which is $1+\epsilon$-equivalent to the unit vector basis of $\ell_{\infty}^{m_{1}}$ and which lies in the linear span of $\left\{y_{n}\right\}_{n=1}^{\infty}$, say, in $\operatorname{span}\left\{y_{1}, \ldots, y_{s_{1}}\right\}$. Let $E_{1}$ be the linear span of $\left\{e_{I}^{1}\right\}_{i=1}^{m_{1}}$ and write $\operatorname{span}\left\{y_{1}, \ldots, y_{s_{1}}\right\}=E_{1}+F_{1}$, where $F_{1}$ is the kernel of a norm $1+\epsilon$ projection from $\operatorname{span}\left\{y_{1}, \ldots, y_{s_{1}}\right\}$ onto $E_{1}$. The vectors $e_{1}^{1}, \ldots, e_{m_{1}}^{1}$ form the first $m_{1}$ terms of our basis.

For the construction of the next $m_{2}$ basis vectors (where $m_{2}$ is appropriately large, depending on $s_{1}$ as well as the sequence $\left.\left\{n_{k}\right\}_{k=1}^{\infty}\right)$, take a subspace $E_{2}$ of the linear span of $\left\{y_{i}\right\}_{i=s_{1}+1}^{s_{2}}$ for some $s_{2}$ so that $E_{2}$ is $1+\epsilon$-isomorphic to the $\ell_{\infty}$ space of its dimension. As long as its dimension is at least $m_{1}$, the space $F_{1}+E_{2}$ is isomorphic to the $\ell_{\infty}$ space of its dimension (which we take to be $m_{2}$ ) with the isomorphism constant depending only on the basis constant of $\left\{y_{n}\right\}_{n=1}^{\infty}$. Let $\left\{e_{i}^{2}\right\}_{i=1}^{m_{2}}$ be a basis for $F_{1}+E_{2}$ which is $d\left(F_{1}+E_{2}, \ell_{\infty}^{m_{2}}\right)$-equivalent to the unit vector basis for $\ell_{\infty}^{m_{2}}$; these vectors form the next $m_{2}$ terms of the desired basis for $X$.

Now just iterate this construction. Let $F_{2}$ be the kernel of a projection of norm $d\left(F_{1}+E_{2}, \ell_{\infty}^{m_{2}}\right)$ from the linear span of $\left\{y_{i}\right\}_{i=s_{1}+1}^{s_{2}}$ onto the linear span of $\left\{e_{i}^{2}\right\}_{i=1}^{m_{2}}$. Then select a subspace $E_{3}$ of the linear span of $\left\{y_{i}\right\}_{i=s_{2}+1}^{s_{3}}$ for some $s_{3}$ so that $E_{3}$ is $1+\epsilon$-isomorphic to the $\ell_{\infty}$ space of its (appropriately large) dimension and continue as in the previous step. It is evident that for any $\left\{n_{k}\right\}_{k=1}^{\infty}$ this construction can produce a basis $\left\{x_{n}\right\}_{n=1}^{\infty}$ which has the property mentioned in the first paragraph of the proof.

Theorem 4.3. The following are equivalent for a Banach space $X$ :

(1) $X$ is a $\mathcal{L}_{p}$ space.

(2) Every compact operator into $X$ factors through some $\mathcal{L}_{p}$ space.

(3) If $T$ is a compact operator from some Banach space $Z$ into $X$ and $Y$ is a separable $\mathcal{L}_{p}$ space, then there are compact operators $A$ from $Z$ into $Y$ and $B$ from $Y$ into $X$ so that $T=B A$ and $B$ is one-to-one.

Remark 4.4. The equivalence of (1) with (2) is known; see [J].

Proof. We show that (1) implies (3). First assume that $X$ is a separable $\mathcal{L}_{p}$ space and let $T: Z \rightarrow X$ be a compact operator; without loss of generality $\|T\| \leq 1$. Take a basis $\left\{x_{n}\right\}_{n=1}^{\infty}$ for $X$ which has the property given in Lemma 4.2. Let $\left\{S_{n}\right\}_{n=1}^{\infty}$ be the partial sum projections associated with $\left\{x_{n}\right\}_{n=1}^{\infty}$ and set $S_{0}=0$. Take $0=n_{0}<n_{1}<\ldots$ so that $\left\|\left(S_{n_{k}}-S_{n_{k-1}}\right) T\right\|<4^{-k}$ for each $k>1$. Now let $Y$ be any separable $\mathcal{L}_{p}$ space and let $\left\{y_{n}\right\}_{n=1}^{\infty}$ be a basis for $Y$ which also has the property given in Lemma 4.2. Thus for each $k$, the spaces span $y_{n_{k-1}+1}, \ldots, y_{n_{k}}$ and span $x_{n_{k-1}+1}, \ldots, x_{n_{k}}$ are isomorphic with the isomorphism constant independent of $k$. Next we use an idea similar to one used in the proof of Lemma 2.4. Take a norm one isomorphism $U_{k}$ from span $y_{n_{k-1}+1}, \ldots, y_{n_{k}}$ onto $\operatorname{span} x_{n_{k-1}+1}, \ldots, x_{n_{k}}$ so that $\sup _{k}\left\|U_{k}\right\|<\infty$. Define $A: Z \rightarrow Y$ by $A z=\sum_{k=1}^{\infty} 2^{k-1} U_{k}^{-1}\left(S_{n_{k}}-S_{n_{k-1}}\right) T$. Let $\left\{P_{n}\right\}_{n=1}^{\infty}$ be the partial sum projections associated with the basis $\left\{y_{n}\right\}_{n=1}^{\infty}$. Define $B: Y \rightarrow X$ by $B y=\sum_{k=1}^{\infty} 2^{-k+1} U_{k}\left(S_{n_{k}}-S_{n_{k-1}}\right) y$. This shows that (1) implies (3).

If $X$ is a non-separable $\mathcal{L}_{p}$ space, we just replace $X$ with any separable $\mathcal{L}_{p}$ subspace which contains the range of $T$ and proceed as above. 
The implication (3) implies (2) is trivial, so we turn to the implication (2) implies (1). First assume $1<p<\infty$, so that every separable $\mathcal{L}_{p}$ space is isomorphic to a complemented subspace of $L_{p}(0,1)$ by $[L P]$. Thus condition (2) implies that every compact operator into $X$ factors through $L_{p}(0,1)$. Now the operators from a space $Z$ into $X$ which factor through $L_{p}(0,1)$ forms a Banach space under the factorization norm $! T !=\inf \|A\| \cdot\|B\|$, where the infimum is over all factorizations $T=B A$ with $A: Z \rightarrow L_{p}(0,1), B: L_{p}(0,1) \rightarrow X$ (use the fact that $L_{p}(0,1)$ is isometric to the $\ell_{p}$ sum of itself). Thus the uniform boundedness principle yields that there is a constant $K=K_{Z}$ so that $! T ! \leq K\|T\|$ for all compact $T$ from $Z$ to $X$. Applying this with $Z$ the $\ell_{1}$ sum of all finite-dimensional subspaces of $X$, we conclude that there is a constant $K$ so that the inclusion from every finite-dimensional subspace of $X$ into $X$ factors through $L_{p}(0,1)$ with factorization constant at most $K$. This implies that $X$ is an $\mathcal{L}_{p}$ space.

This argument does not quite work for $p=1$ or $p=\infty$ since not every separable $\mathcal{L}_{1}$ space is isomorphic to a complemented subspace of $L_{1}(0,1)$, and no infinite-dimensional separable $\mathcal{L}_{\infty}$ space is isomorphic to a complemented subspace of $L_{\infty}[0,1]$. Here is how to get around that annoyance. The argument in $[L P]$ yields that the injection $J_{Y}$ from a separable $\mathcal{L}_{p}$ space into its second dual factors through $L_{p}(0,1)$ in the extreme case $p=1$. When $p=\infty$, the same is true by the injectivity of the second dual of an $\mathcal{L}_{\infty}$ space. The argument in the preceding paragragh then yields that the inclusion from every finite-dimensional subspace of $X$ into $X^{* *}$ factors through $L_{p}(0,1)$ with factorization constant at most $K$. This is enough, by $\left[\mathrm{LR}\right.$, to guarantee that $X$ is an $\mathcal{L}_{p}$ space.

Remark 4.5. It is evident that condition (3) in Theorem 4.3 is equivalent to:

(4) For every compact subset $K$ of $X$ and every separable $\mathcal{L}_{p}$ space $Y$ there is a one-to-one compact operator $S$ from $Y$ to $X$ so that $S\left(B_{Y}\right) \supset K$.

That the one-to-oneness is essential in Remark 4.5 is given by the following proposition.

Proposition 4.6. Let $1 \leq p<\infty$. The following are equivalent for a Banach space $X$.

(5) For every compact subset $K$ of $X$ and every $\mathcal{L}_{p}$ space $Y$ there is a compact operator $S$ from $Y$ to $X$ so that $S\left(B_{Y}\right) \supset K$.

(6) For every compact subset $K$ of $X$ there is an $\mathcal{L}_{p}$ space $Y$ and an operator $S$ from $Y$ to $X$ so that $S\left(B_{Y}\right) \supset K$.

(7) $X$ is isomorphic to a quotient of $L_{p}(\mu)$ for some measure $\mu$.

Proof. The case $p=1$ is easy because every Banach space is a quotient of $\ell_{1}(\Gamma)$ for some set $\Gamma$ and every $\mathcal{L}_{1}$ space contains a complemented copy of $\ell_{1}$. So assume that $1<p<\infty$. If condition (6) holds, then the space $Y$ can always be taken separable and hence $Y$ can be taken to be $L_{p}(0,1)$ because every separable $\mathcal{L}_{p}$ space is isomorphic to a complemented subspace of $L_{p}(0,1)$.

We claim that there is a constant $C$ so that every compact subset of the unit ball of $X$ is covered by $S\left(B_{L_{p}(0,1)}\right)$ for some operator $S$ from $L_{p}(0,1)$ into $X$ for which $\|S\| \leq C$. Indeed, otherwise there would be compact subsets $K_{n} \subset B_{X}$ so that if $K_{n} \subset S\left(B_{L_{p}(0,1)}\right)$ with $S: L_{p}(0,1) \rightarrow X$, then $\|S\|>n^{2}$. Set $K=\sup _{n=1}^{\infty} n^{-1} K_{n} \cup\{0\}$. Then $K$ is a compact subset of $B_{X}$ and so there is an operator $S: L_{p}(0,1) \rightarrow X$ 
so that $K \subset S\left(B_{L_{p}(0,1)}\right)$. Then $(n S)\left(B_{L_{p}(0,1)}\right) \supset K_{n}$ and so $\|n S\| \geq n^{2}$ for all $n$. This proves the claim.

By the claim, there is a $C$ so that for each finite-dimensional subspace $E$ of $X$ there is an operator $S_{E}: L_{p}(0,1) \rightarrow X$ so that $S_{E} B_{L_{p}(0,1)} \supset B_{E}$ and $\left\|S_{E}\right\| \leq C$. Direct the finite-dimensional subspaces of $X$ by inclusion and let $\mathcal{U}$ be any ultrafilter on this directed set which contains all tails $T_{E}:=\{F: E \subset F \subset X ; \operatorname{dim} F<\infty\}$, where $E$ ranges over the finite-dimensional subspaces of $X$. It is easily checked that the weak* operator limit through $\mathcal{U}$ of $S_{E}^{*}$ is an isomorphism from $X^{*}$ into the ultrapower $L_{q}(0,1)_{\mathcal{U}}$, where $1 / p+1 / q=1 . L_{q}(0,1)_{\mathcal{U}}$ is isometrically isomorphic to $L_{q}(\mu)$ for some measure $\mu$, so we now know that $X$ is reflexive and thus $X^{*}$ is separable. This implies that $X^{*}$ is isomorphic to a subspace of $L_{q}(0,1)$ and hence $X$ is isomorphic to a quotient of $L_{p}(0,1)$. This completes the proof that condition (6) implies condition (7).

Finally we assume that (7) is satisfied. Let $Q$ be a quotient map from $L_{p}(\mu)$ onto $X$. If $K$ is a compact subset of $X$, then it is easy to see that some superspace of $K$ in $X$ is the image of some separable $L_{p}$ subspace of $L_{p}(\mu)$ and hence of $L_{p}(0,1)$. That is, without loss of generality we can assume that $X$ is separable and that $L_{p}(\mu)$ is $L_{p}(0,1)$. Now by Michael's selection theorem (or the Bartle-Graves theorem) there is a compact subset $K_{1}$ of $L_{p}(0,1)$ so that $Q K_{1}=K$. By Lemma 2.4. there is a compact operator $T$ on $L_{p}(0,1)$ so that $T B_{L_{p}(0,1)} \supset K_{1}$. Then $S:=Q T$ is a compact operator from $L_{p}(0,1)$ into $X$ for which $S B_{L_{p}(0,1)} \supset K$. This completes the proof of Proposition 4.6.

We have frequently used the fact that every compact subset of a Banach space is contained in the closed convex symmetric hull of a sequence which converges to zero. What are the Banach spaces for which the null sequence can always be chosen to have some extra property such as being basic or at least countably linearly independent? It is a good exercise for students to show that Hilbert space does not have one of these stronger properties. A complete answer to the question is given by the next theorem.

Theorem 4.7. The following are equivalent for a Banach space $X$ :

(1) $X$ is a $\mathcal{L}_{1}$ space.

(2) For each compact subset $K$ of $X$ there is a basic sequence which converges to zero and whose closed convex symmetric hull contains $K$.

(3) For each compact subset $K$ of $X$ there is a sequence $\left\{y_{n}\right\}_{n=1}^{\infty}$ which converges to zero and whose closed convex symmetric hull contains $K$ and such that $0 \neq$ $\sum_{n=1}^{\infty} \lambda_{n} y_{n}$ whenever $0<\sum_{n=1}^{\infty}\left|\lambda_{n}\right|<\infty$.

If $X$ is separable, the preceding conditions are equivalent to

(4) For each compact subset $K$ of $X$ there is a basis for $X$ which converges to zero and whose closed convex symmetric hull contains $K$.

Proof. Assume that (1) holds and $K$ is a compact subset of $B_{X}$. Let $Y$ be a separable $\mathcal{L}_{1}$ subspace of $X$ which contains $K$ and let $\left\{x_{n}\right\}_{n=1}^{\infty}$ be a basis for $X_{0}$ which satisfies the conclusion of Lemma 4.2, Let $\left\{S_{n}\right\}_{n=1}^{\infty}$ be the partial sum projections associated with $\left\{x_{n}\right\}_{n=1}^{\infty}$ and set $S_{0}=0$. From Lemma 2.4 we get $0=n_{0}<n_{1}<\ldots$ so that image of $B_{X}$ under the operator

$$
T:=\sum_{k=1}^{\infty} 4^{-k+1}\left(S_{n_{k}}-S_{n_{k-1}}\right)
$$


contains $K$. By the property of the sequence $\left\{x_{n}\right\}_{n=1}^{\infty}$, for each $k$ there is a basis $z_{n_{k-1}}, \ldots, z_{n_{k}}$ for the linear span $E_{k}$ of $x_{n_{k-1}}, \ldots, x_{n_{k}}$ so that the unit ball of $E_{k}$ is contained in the convex symmetric hull of $z_{n_{k-1}}, \ldots, z_{n_{k}}$ and such that $\sup _{1 \leq n<\infty}\left\|z_{n}\right\|:=C<\infty$. Let $C_{1}:=\sup _{k}\left\|S_{n_{k}}-S_{n_{k-1}}\right\|$. From the property of the operator $T$ we deduce that $K$ is contained in the closed convex symmetric hull of the sequence $y_{n}$, where $y_{j}=2 C C_{1} 2^{-k+1} z_{j}$ if $n_{k-1}<j \leq n_{k}$. This completes the proof that (1) implies (2) and that (1) implies (4) when $X$ is separable. The only remaining non-trivial implication is that (3) implies (1). But (3) implies that every compact set in $X$ can be covered by the image of the unit ball of $\ell_{1}$ under some one-to-one operator from $\ell_{1}$ into $X$, so $X$ is an $\mathcal{L}_{1}$ space by condition (4) in the remark after Theorem 4.3 .

\section{REFERENCES}

[BDGJN] G. Bennett, L. E. Dor, V. Goodman, W. B. Johnson, and C.M. Newman, "On uncomplemented subspaces of $L_{p}, 1<p<2$," Israel J. Math. 26 (1977), 178-187. MR 0435822 $(55: 8778)$

[F] T. Figiel, "Factorization of compact operators and applications to the approximation problem," Studia Math. 45 (1973), 191-210. MR0336294 (49:1070)

[FJ] T. Figiel and W. B. Johnson, "The approximation property does not imply the bounded approximation property," Proc. Amer. Math. Soc. 41 (1973), 197-200. MR0341032 $(49: 5782)$

[Fo1] V. P. Fonf, "One property of families of embedded Banach spaces," J. Soviet Math. 59 (1992), 687-690. MR.1157744 (93e:46014)

[Fo2] V. P. Fonf, "On the extension of operator bases in Banach spaces," Teor. Funkt., Funkt. Anal. i Prilozh. 54 (1990), 37-42 (Russian), English transl. in Soviet Math. 1991, 319322. MR1080722 (92d:46026)

[FJPP] V. P. Fonf, W. B. Johnson, G. Pisíer, and D. Preiss, "Stochastic approximation properties in Banach spaces," Studia Math. 159 (2003), no. 1, 103-119. MR2030905 (2004k:46011)

[GL] Y. Gordon and D. R. Lewis, "Absolutely summing operators and local unconditional structures," Acta Math. 133 (1974), 27-48. MR0410341 (53:14091)

[He] W. Herer, "Stochastic bases in Frechet spaces," Demonstratio Math. 14 (1981), 719724. MR0663121 (83k:46012)

[J] W. B. Johnson, "Factoring compact operators," Israel J. Math. 9 (1971), 337-345. MR0290133 (44:7318)

[JRZ] W. B. Johnson, H. P. Rosenthal, and M. Zippin, "On bases, finite-dimensional decompositions and weaker structures in Banach spaces," Israel J. Math. 9 (1971), 488-506. MR0280983(43:6702)

[LR] J. Lindenstrauss and H. P. Rosenthal, "The $\mathcal{L}_{p}$ spaces," Israel J. Math. 7 (1969), 325349. MR0270119(42:5012)

[LT] J. Lindenstrauss and L. Tzafriri, "Classical Banach Spaces I," Springer-Verlag, 1977. MR0500056 (58:17766)

[NW] N. J. Nielsen and P. Wojtaszczyk, "A remark on bases in $\mathcal{L}_{p}$-spaces with an application to complementably universal $\mathcal{L}_{\infty}$-spaces," Bull. Acad. Polon. Sci., Sér. Sci. Math. Astronom. Phys. 21 (1973), 249-254. MR0322484 (48:846)

[P1] A. Pełczyński, "Any Banach space with the bounded approximation property is a complemented subspace of a Banach space with a basis," Studia Math. 40 (1971), 239-242. MR0308753 (46:7867)

[P2] A. Pełczyński, "Universal bases," Studia Math. 32 (1969), 247-268. MR0241954 (39:3290)

[PR] A. Pełczyński and H. P. Rosenthal, "Localization techniques in $L_{p}$ spaces," Studia Math. 52 (1974/75), 263-289. MR0361729 (50:14174)

[Pl] A. M. Plichko, "The choice of subspaces with special properties in a Banach space and some properties of quasi-complements," Functional Anal. and Appl. 15 (1981), 88-89 (Transl. from Russian). MR0609803 (82e:46031) 
[Sc] G. Schechtman, "On Pełczyński's paper "Universal bases," " Israel J. Math. 22 (1975), 181-184. MR0390730 (52:11553)

[Sz] S. J. Szarek, "A Banach space without a basis which has the bounded approximation property," Acta Math. 159 (1987), 81-98. MR0906526 (88f:46029)

[T] P. Terenzi, "A complement to Krein-Milman-Rutman theorem with applications," Instituto Lombardo (Rend. Sc.) A 113 (1979), 341-353. MR0622113 (82h:46022)

Department of Mathematics, Ben-Gurion University of the Negev, P.O. Box 653, Beer-Sheva 84105, Israel - and - Department of Mathematics, Texas A\&M University, College Station, Texas 77843

E-mail address: fonf@black.bgu.ac.il

Department of Mathematics, Texas A\&M University, College Station, Texas 77843

E-mail address: johnson@math.tamu.edu

Instytut Matematyki, Politechnika Krakowska im. Tadeusza Kosciuszki, ul. WarSZAWSka 24, Krakow 31-155, Poland

E-mail address: aplichko@usk.pk.edu.pl

Sebastian-Kneipp Gasse, 7, Augsburg 86152, Germany

E-mail address: vshevchyk@hotmail.com 